Review

\title{
Adaptive Video Quality Model for UHD Video Broadcasting Using the Principle of Inclusion
}

\author{
Urvashi Pal and Horace King \\ College of Engineering and Science, Victoria University, Melbourne, Australia
}

\section{Article history}

Received: $24-08-2017$

Revised: 25-10-2017

Accepted: 18-11-2017

Corresponding Author:

Urvashi Pal

College of Engineering and

Science, Victoria University,

Melbourne, Australia

Email: Urvashi.pal@live.vu.edu.au

\section{Introduction}

The future of video broadcasting not just lies in resolution, but also in new technologies like High Frame Rate (HFR), Wide Colour Gamut (WCG), High Efficiency Video Coding (HEVC) and more. These video parameters contribute towards the ultimate viewing experience (Vignelles and Marshall, 2015). However, the availability of required resources for the bandwidth hungry Ultra High Definition (UHD) video will be a challenge that the broadcasters need to overcome. In such a scenario, allocating dedicated resources to maintain a fixed quality of video will result in the wastage of resources or video outage, in case the resources are not enough to deliver the promised quality. An adaptive video quality model using the principle of inclusion as given in this study, resolves this problem by efficiently allocating the available resources (channel capacity, coverage area, distance between transmitter and receiver and separation distance), by trading off the video quality.

\section{UHD Video Parameters}

\section{Resolution}

UHDTV has a resolution of $3840 \times 2160$ pixels, which is four times the resolution of HDTV. This means that there is four times more information displayed on screen, which is one of the factors to enhance the video quality (Eutelsat, 2015).

\section{High Frame Rates (HFR)}

Frame rate used till now for HD is $25 \mathrm{fps}$ (frames per second), but for UHD we will be dealing with $50 \mathrm{fps}$, $100 \mathrm{fps}$ or even higher. Increasing the frame rate increases the smoothness of a video, especially for high motion contents (Limmer and Chabrol, 2014). In other words, increased information per second of the video with more frames enhances the smoothness and colour rendition (Pal and King, 2015a).

\section{Higher Dynamic Range (HDR), WCG \& Rec. 2020}

UHD technology allows for a greater array of colours to be perceived by viewers. Rec. 709 captures $35 \%$ of the natural view, while Rec.2020 captures $75 \%$. The wide range of colours is going to radically enhance the picture quality of a UHD video (Kenichiro et al., 2014).

\section{Video Compression}

At present, MPEG-4 video compression format is being used to watch HD channels on our HDTVs. HEVC is the new video compression method, developed especially to compress the huge data of UHD and has been adopted for its transmission by DVB (Advanced Television, 2014). HEVC offers 50\% higher video compression and quality as compared to MPEG-4 and therefore, will make the transmission of UHD content more efficient by saving the bandwidth significantly. 
However, to use HEVC, broadcasters will have to invest in upgraded infrastructure, which will take time and cost a lot of money.

\section{Television Size}

The ideal size of a UHDTV is supposed to be around $55 "$ to 80 ". Based on the size of television, viewing distance is calculated to maintain the maximum perceived angular resolution because there are limits to what an eye can perceive (TT, 2013). If you sit too close to the TV, you will be seeing the unwanted individual pixels and if you sit too far, you won't be able to observe all the details in the video. That means, if you sit too far away from a UHDTV, the UHD content will look like HD. As a result, the viewing distance for a UHDTV is half of what is required for HDTV.

\section{Problem Formulation}

For a successful transmission and reception of a UHD video, it is important that every block in the broadcast chain must be upgraded. This will lead to an overall increase in the cost of production and broadcasting but the enhanced video quality with richer colours and dynamic motion range makes the effort totally worth it (Pal and King, 2017). Still, at the moment, broadcasters will opt for a trade off in video quality by artificially upscaling a lower resolution content rather than using the original high resolution content in the initial phase of broadcasting (Intelsat, 2015).

The availability of numerous options to select from for a UHD and HD video will itself create confusion in the future broadcast scenario for the Direct to Home (DTH) operators. It is also important that advanced hardwares support interoperability at every stage, which will take time. Many video standards with varying resolutions, frame rates and compression, as depicted in Fig. 1, will have to support future transmissions (SES, 2013). Therefore, an adaptive stream methodology is proposed in this study, which uses a statistical approach to assign the best signal stream as per the reception criteria.

\section{Experimental Model and Results}

The following experiment is performed to understand the signal behaviour in a broadcast communication channel. Using the BER vs. SNR results, its correlation with the channel capacity, separation distance and coverage area is analysed. Based on this analysis, a statistical adaptive video quality algorithm is developed for UHD video broadcasting through DVB-S2, using the principle of inclusion.

\section{DVB-S2 Model}

Using the generic DVB-S2 model, as explained in detail in (Pal and King, 2015b), information bits are extracted from a UHD video and transmitted through the MATLAB built DVB-S2 model, as given in Fig. 2
(DVB, 2005). The results achieved are for a Rician Fading Channel, at $\mathrm{K}=5$ and $\mathrm{SNR}=20 \mathrm{~dB}$. The noise channel consists of a Rician Fading Channel, Correlated Phase Noise and AWGN. BER vs. SNR graph is generated for a range of modulation and coding schemes, as given in Fig. 3.

\section{BER vs. SNR Results}

The results show that for a Rician Fading Channel $(\mathrm{K}=5)$, BER decreases to $10^{-6}$ level for most of the MODCOD schemes, except 32APSK, which is a complex modulation scheme to be decoded successfully in the presence of heavy noise.

\section{Proposed Modeling Using Experimental Results}

Correlation of Channel Capacity and BER vs. SNR Results

Using Shannon Capacity Theorem and SNR results from the above experiment, capacity of the channel is calculated using Equation 1 and plotted against its BER values, in Fig. 4:

$$
\frac{C}{B}=\log _{2}\left(1+\frac{S}{N}\right)
$$

\section{Where:}

$$
\begin{aligned}
& C=\text { Capacity of the channel in bits/second } \\
& B=\text { Bandwidth of the channel in Hertz } \\
& S=\text { Signal power in Watts } \\
& N=\text { Noise power in Watts } \\
& C / B=\text { Bits/seconds/hertz }
\end{aligned}
$$

Figure 4 shows that the maximum capacity of a channel is reached at $10^{-6}$ for a Rician Fading Channel. Also, the maximum capacity is reached earlier by 32APSK and 16APSK, as compared to 8PSK and QPSK. This shows that, even though M-PSK has a lower symbol rate than M-APSK, its probability of error is also low. Therefore, more reliable information can be transmitted though M-PSK than M-APSK.

\section{Coverage Area: Distance between Transmitter and Receiver}

The link budget model according to Friss-Free-Space Path Loss formula is:

$P_{r}=P_{t}+G_{t}+G_{r}-P_{L}$

Where:
$P_{t} \quad=$ Transmit power
$P_{r} \quad=$ Received power at distance ' $\mathrm{d}$ '
$G_{t}$ and $G_{r}=$ Antenna gain for transmit and receive antennas respectively (both assumed to be $0 \mathrm{~dB}$ for simplicity) 


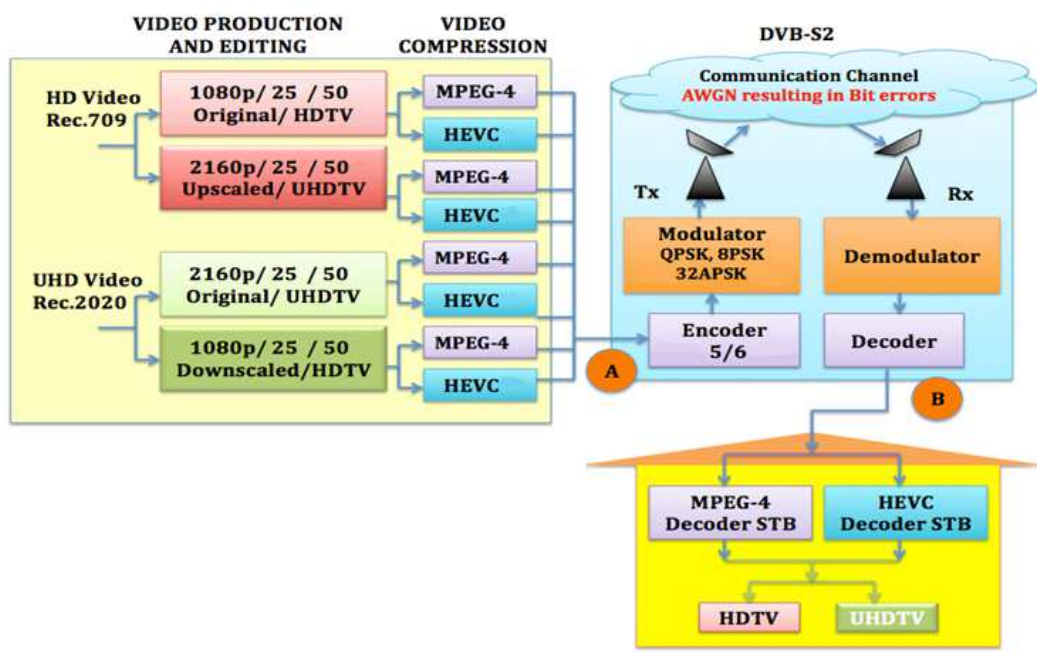

Fig. 1: Co-existence of multiple video standards

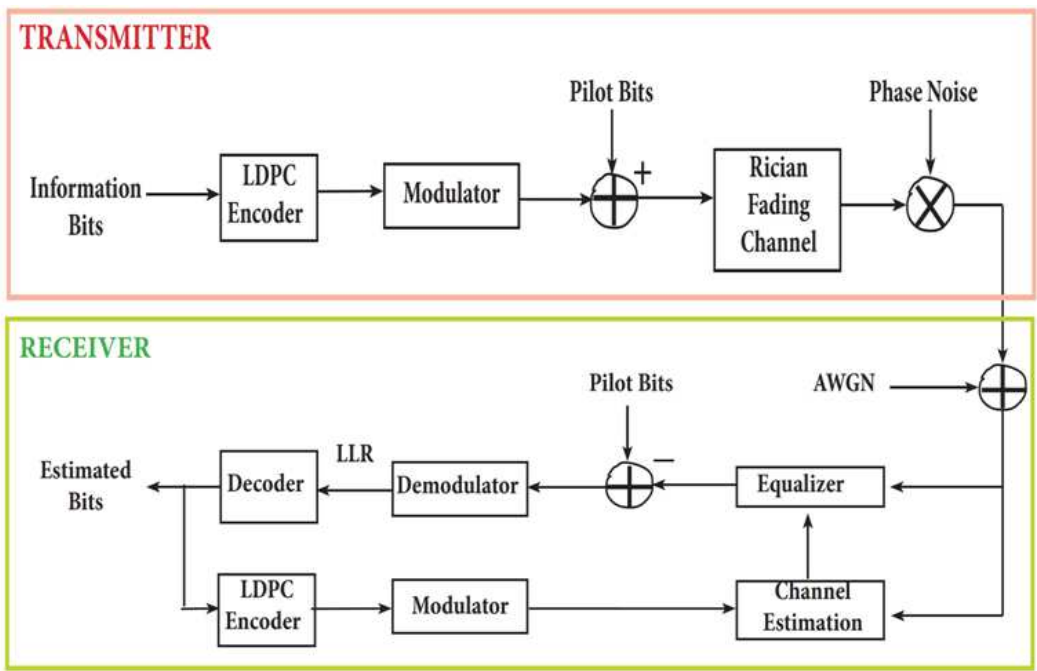

Fig. 2: DVB-S2 block schematic

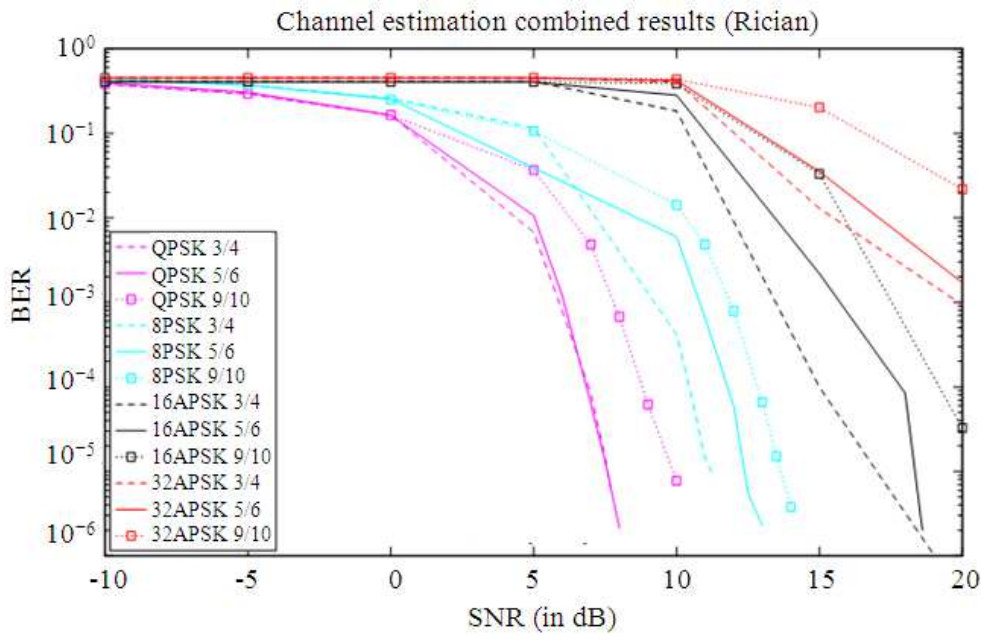

Fig. 3: BER vs. SNR graph 


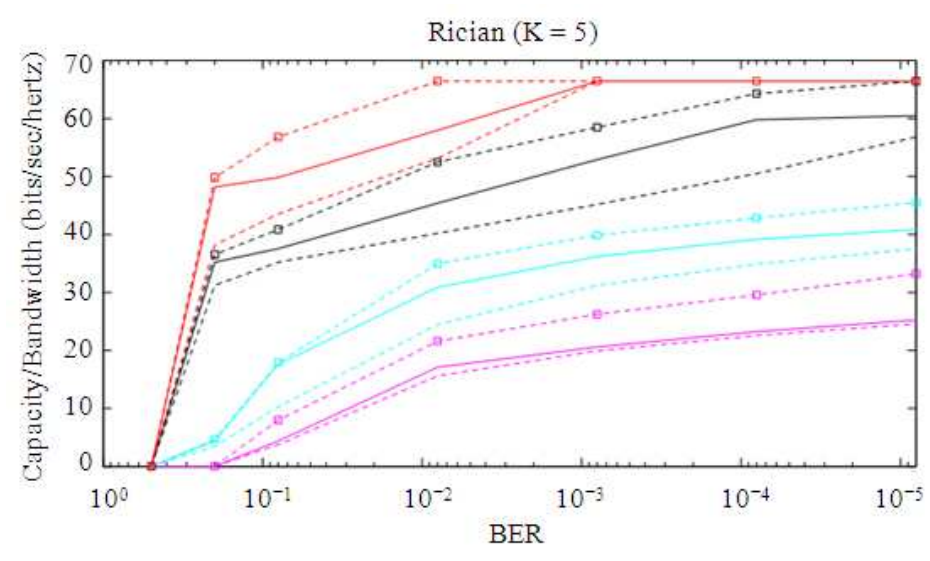

Fig. 4: Capacity vs. BER graph for rayleigh and rician fading channel (legend same as Fig. 3.)

The received signal strength is dominated by the distance from the transmitter and the receiver and the general path loss model can be expressed as in Equation 3 where ' $\lambda$ ' is the wavelength corresponding to the center frequency $f_{c}$ and ' $n$ ' is the path loss exponent which can be approximated as 2 (Wang and Zhang, 2012):

$$
P_{L}(d B)=10 \log _{10}\left(\frac{4 \pi d}{\lambda}\right)^{n}
$$

Suppose, frequency range from 57 to $64 \mathrm{GHz}$ is being used, the constraint on transmit power is $P_{t} \leq 40 \mathrm{dBm}$. If thermal noise is the primary source of interference, the required sensitivity $\left(S_{r}\right)$ at the receiver can be calculated as:

$S_{r}=N F+F+S N R$

Where:

$N F=$ The noise floor calculated by thermal noise: $\mathrm{N}$ $=\mathrm{kTWF}$

$F \quad=$ The noise figure (optimistically) assumed to be $0 \mathrm{~dB}$

$S N R=$ The Signal to Noise Ratio at the receiver

$k=$ Boltzmann's constant

$T=$ The room temperature (typically $290 \mathrm{~K}$ )

For the $60 \mathrm{GHz}$ systems, the noise floor is calculated as $-76 \mathrm{dBm}$. To ensure adequate performance at the receiver, the minimum received power should be greater than or equal to the required sensitivity as expressed in Equation 5:

$$
S N R \leq 116-\log _{10}\left(\frac{4 \pi d}{\lambda}\right)^{2}
$$

Channel capacity can be calculated according to the Shannon capacity and the relationship between the capacity and communication distance is then given by:

$C \leq B \log _{2}\left(1+10^{116-10 \log _{10}\left(\frac{4 \pi d}{\lambda}\right)^{n} / 10}\right)$ taking into account the contribution by $S N R$ in Equation 5.

Substituting the values of Shannon Capacity ' $C$ ' from Equation 1 into Equation 6, ' $d$ ' is calculated. Using SNR values from the above experiment, we plot Distance ' $d$ ' between the Transmitter and Receiver vs. BER graph for Rician Fading Channel.

The results in Fig. 5 show that as ' $d$ ' decreases, Signal strength increases and errors decrease. (Values assumed: $n=2, \lambda=10, \pi=3.14$ ).

\section{Analysis of Service Area Separation Distance}

Spectrum efficiency is a function of the size of the broadcaster's coverage area and the separation distance between these coverage areas. To obtain the maximum achievable efficiency of spectrum use, which is a function of both the size of the broadcaster's coverage area and the distance separating them, broadcasters are packed in a regular hexagonal constellation, as shown in Fig. 6, to achieve the highest average density of broadcasters on a per area basis (Bettancourt and Peha, 2015). Consider a statistical path loss model where the median path loss depends only on the distance from each transmitter. For a traditional broadcaster, a circle in the hexagon represents the interference-limited coverage area, centred at the transmitter, with radius $R_{\text {trad }}$ equal to the distance between the transmitter and the nearest point on the edge of the coverage area. Where, $C_{\text {trad }}$ is the minimum distance between coverage areas of two traditional broadcasters.

The maximum fraction of area that can be covered by traditional broadcasters divided by the area of their respective hexagonal tile in the lattice, is given by:

$$
\eta=\frac{R_{\text {trad }}^{2}}{\left(R_{\text {trad }}+0.5 C_{\text {trad }}\right)^{2}} \cdot \frac{\pi}{2 \sqrt{3}}
$$

Where:

$\eta \quad=$ Spectral Efficiency (Modulation Efficiency of the MODCOD scheme)

$R_{\text {trad }}=$ Distance between transmitter and receiver

$C_{\text {trad }}=$ Separation distance between two coverage areas 


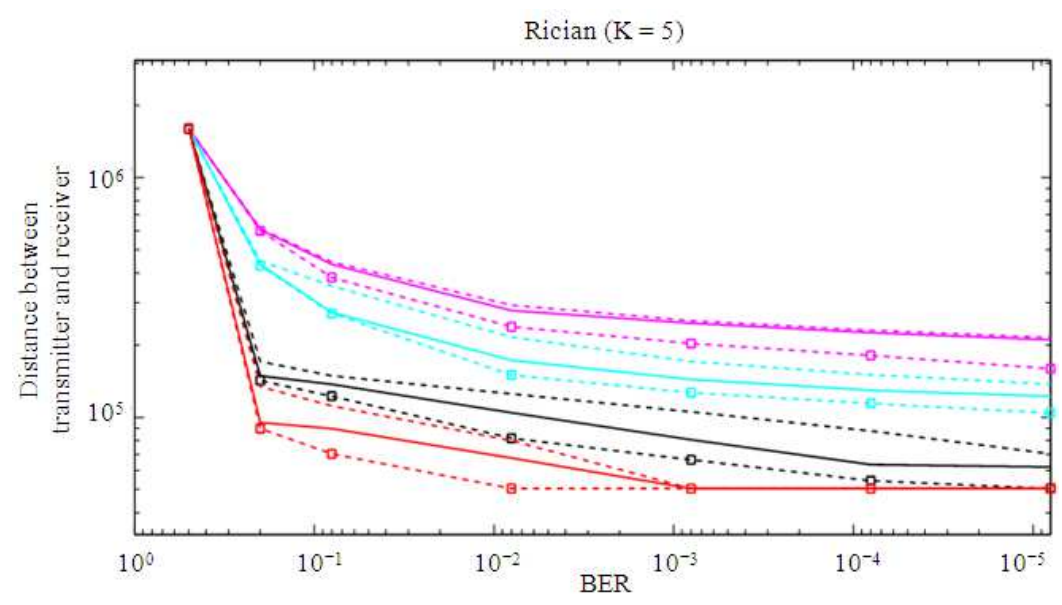

Fig. 5: Distance between transmitter and receiver vs. BER for Rician (legend same as Fig. 3.)

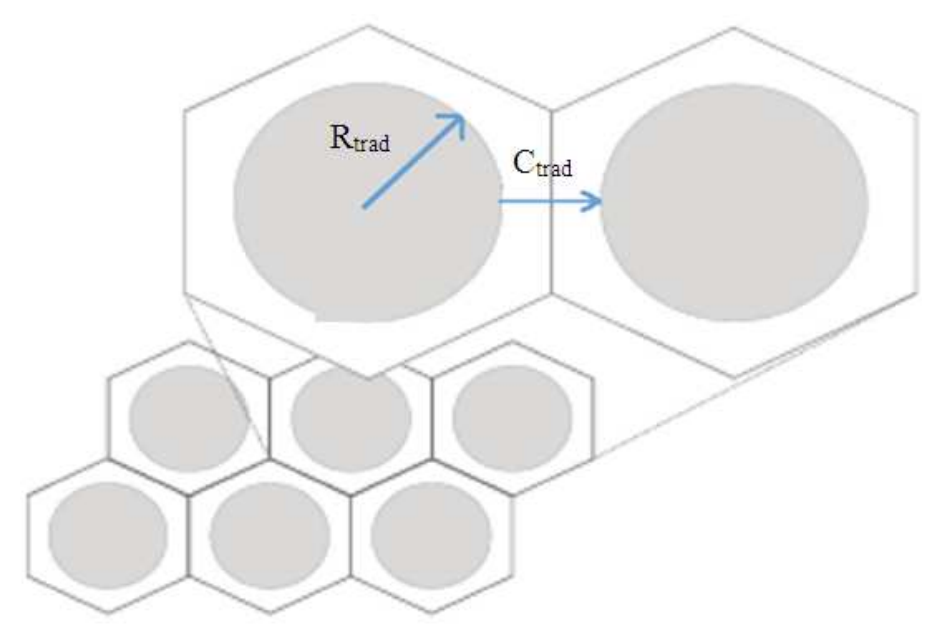

Fig. 6: Hexagonal packing of co-channel traditional broadcasters

Substituting the values of spectral efficiency (DVB, 2005) and distance between transmitter and receiver from the previous section, in Equation $7, C_{\text {trad }}$ is calculated and plotted again the BER.

As the distance between the transmitter and receiver increases, required transmit power to maintain a low BER increases. As the transmit power increases, the coverage area increases and the separation distance between two coverage areas decreases. When the separation distance is high, error probability from the adjacent coverage area is low. But when the separation distance is small, noise is high and coverage area is small.

Large coverage areas require larger separation distance to maintain low interference from adjacent cells. Therefore, there is a trade-off between transmit power and noise as spectrum efficiency increases with coverage area and decreases with separation distance. Hence, the larger the coverage area, the lower the spectrum efficiency. As a result, it is efficient in terms of spectrum efficiency to provide TV service to a given area by using many small individual coverage areas rather than few large coverage areas. The graph for separation distance vs. BER is plotted in Fig. 7, which shows that as the separation area decreases, BER or noise increases.

\section{Applying the Principle of Inclusion}

Suppose, number of cells in active set $\leq 4$; respectively represented by $b_{1}, b_{2}$ and $b_{3}$. Let ' $\mathrm{K}$ ' be a set with $|K|=Z$ in service area $J$ and let $b_{1}, b_{2} \ldots b_{t}$ be a collection of conditions, such as Coverage area, Distance between transmitter and receiver and Separation distance, satisfied by some or all of the elements of ' $K$ '. Some elements of ' $K$ ', such as $S N R, B E R$ and Capacity, may satisfy more than one of the conditions, whereas others may not satisfy any of them (King and Pal, 2015).

Denote the number of elements in ' $K$ ' that satisfy condition bi for $1 \leq i \leq \mathrm{t}$ by $Z\left(b_{i}\right)$. Elements of ' $K$ ' are only valid when they satisfy only condition $b_{i}$ as well as 
when they satisfy other conditions $b_{j}$ for $j \neq i$. Therefore for any $i, j \in 1,2,3, \ldots, t$ where $j \neq i Z\left(b_{i} b_{j}\right)$ denotes the number of elements in $K$ that satisfy both of the conditions $b_{i}$ and $b_{j}$. If $1 \leq i, j, k \leq t$ are three distinct values, then $\left(b_{\mathrm{i}} b_{\mathrm{j}} b_{\mathrm{k}}\right)$ denotes the number of elements in ' $K$ ' satisfying each of the conditions $b_{i}, b_{j}$ and $b_{k}$. Therefore, for each $1 \leq i \leq t, Z\left(b_{1}^{\prime}\right)=Z-Z\left(b_{i}\right)$ will denote the number of elements in ' $K$ ' that do not satisfy condition $b_{i}$. However, if $1 \leq i, j \leq t$ with $i \neq j,\left(b_{i}^{\prime} b_{j}^{\prime}\right)$ equates to the number of elements in ' $K$ ' that do not satisfy either of the conditions $b_{i}$ or $b_{j}$. Hence:

$$
Z\left(b_{i}^{\prime} b_{j}^{\prime}\right)=Z-\left[Z\left(b_{i}\right)+Z\left(b_{j}\right)+Z\left(b_{i} b_{j}\right)\right.
$$

The 3rd term in Equation 8 is added because it is eliminated twice in the second term $\left[Z\left(b_{i}\right)+Z\left(b_{j}\right)\right]$. From Equation 8, it is possible to determine the number of elements of ' $K$ ' that satisfy none of the conditions $b_{i}$, for $1 \leq i \leq t$. This is denoted by $Z^{\prime}=Z\left(b_{1}^{\prime} b_{2}^{\prime} b_{3}^{\prime} \ldots b_{t}^{\prime}\right)$ and by expansion:

$$
\begin{aligned}
& Z^{\prime}=Z-\sum_{1 \leq i \leq t} Z\left(b_{i}\right)+\sum_{1 \leq j \leq t} Z\left(b_{i} b_{j}\right) \\
& -\sum_{1 \leq i<j<k \leq t} Z\left(b_{i} b_{j} b_{k}\right)+\ldots . .+(-1)^{t} Z\left(b_{1} b_{2} b_{3} \ldots . . b_{t}\right)
\end{aligned}
$$

Using Equation 9 for ' $s$ ' $\in K$ and that ' $s$ ' satisfies none of the conditions in Equation 9; it is clear that ' $s$ ' is counted once in $Z$ ' and once in $Z$ but will not be counted in any of the other three terms in Equation 9. It is evident that the number of elements in ' $K$ ' that satisfy at least one of the conditions $b_{\mathrm{i}}$ where $1 \leq i \leq t$ is given by $Z$ ( $b_{1}$ or $b_{2}$ or $\ldots$ or $\left.b_{t}\right)=Z-Z$ '. The following notation further simplifies Equation 9 such that:

$$
\begin{aligned}
& \left.K_{1}=Z\left(b_{1}\right)+Z\left(b_{2}\right)+\ldots+Z\left(b_{t}\right)\right] \\
& K_{k}=\left[\sum Z\left(b_{i_{1}} b_{i_{2}} b_{i_{k}}\right)\right], 1 \leq k \leq t(t)
\end{aligned}
$$

The summation in Equation 10 is taken overall selections of size ' $k$ ' from the collection of ' $t$ ' conditions and $K_{k}$ has $\left(\begin{array}{l}t_{J} \\ k_{J}\end{array}\right)$ summands in it.

Equation 9 and 10 can be used to establish whether all the conditions that enhance the video quality are met. If one of the conditions is not met then the user cannot view a video having the best quality parameters. This may mean a change in video parameters to the active set or may necessitate requiring more resources to be allocated.

\section{Proposed Adaptive Video Quality Model for UHD Video Broadcasting}

In Table 1, the best-case scenario is represented by case 1, where the coverage area is small, separation distance is big and the distance between transmitter and receiver is also small. Due to these factors, it is possible to achieve the BER of $10-6$ at a SNR $\geq 6 \mathrm{~dB}$. Therefore, the capacity consumed is $\leq 75 \%$. As a result of these conditions, the video quality viewed on TV has a resolution and frame rate of $2160 \mathrm{p} / 50$, colour profile of Rec.2020, with HEVC codec. Such a video must be viewed on TV screen of size $\geq 55$ ". However, as the conditions vary, the resultant video quality also varies, as given in the table below.

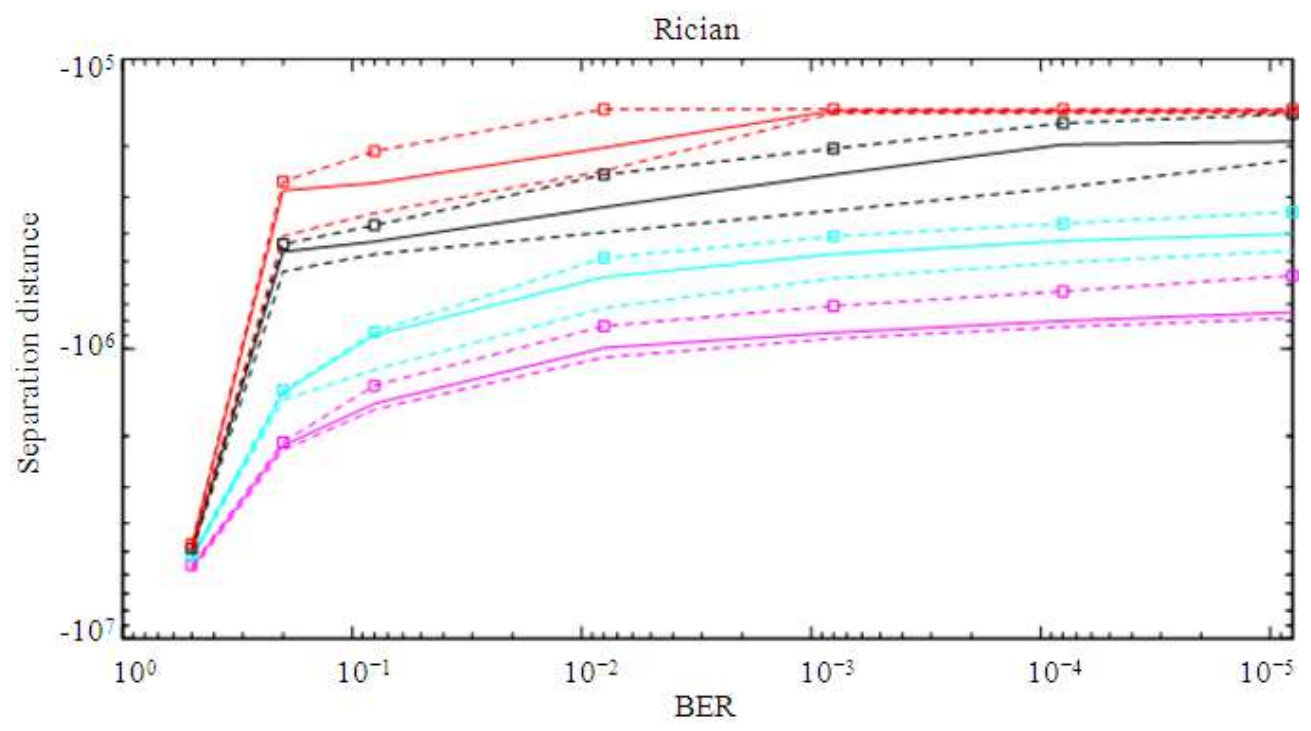

Fig. 7: Separation distance vs. BER graph for Rician (legend same as Fig. 3) 
Table 1: Video quality result in different scenarios applying the principle of inclusion

\begin{tabular}{|c|c|c|}
\hline & Scenario & Video result \\
\hline \multirow[t]{6}{*}{$\overline{1}$} & Coverage area $=$ Low & Res/fps $=2160 \mathrm{p} / 50$ \\
\hline & Separation distance $=$ High & Colour $=$ Rec2020 \\
\hline & $\mathrm{d}=\mathrm{Low}$ & Codec $=$ HEVC \\
\hline & $\mathrm{BER}=10^{-6}$ & Ideal TV Size $\geq 55$ ” \\
\hline & $\mathrm{SNR} \geq 6 \mathrm{~dB}$ & Best video quality using \\
\hline & Capacity $\leq 75 \%$ & future resources \\
\hline \multirow[t]{6}{*}{2} & Coverage area $=$ Low & \\
\hline & Separation distance $=$ Low & $\operatorname{Res} / f p s=1080 / 50 p$ \\
\hline & $\mathrm{d}=$ Low & Colour $=$ Rec 2020 \\
\hline & $\mathrm{BER}=10^{-6}$ & Codec $=$ HEVC \\
\hline & $\mathrm{SNR} \geq 6 \mathrm{~dB}$ & Ideal TV Size $=45-55 ”$ \\
\hline & Capacity $\leq 75 \%$ & Using many resources \\
\hline \multirow[t]{6}{*}{3} & Coverage area $=$ Low & \\
\hline & Separation distance $=$ Low & $\operatorname{Res} / \mathrm{fps}=1080 / 25 \mathrm{p}$ \\
\hline & $\mathrm{d}=$ High & Colour $=$ Rec709 \\
\hline & $\mathrm{BER}=10^{-5}$ & Codec $=$ MPEG -4 \\
\hline & $\mathrm{SNR} \geq 6 \mathrm{~dB}$ & Ideal TV Size $=40-50 ”$ \\
\hline & Capacity $\leq 75 \%$ & Using available resources \\
\hline \multirow[t]{6}{*}{4} & Coverage area $=$ High & Res $/ f p s=1080 / 25 i$ \\
\hline & Separation distance $=$ Low & Colour $=$ Rec 709 \\
\hline & $\mathrm{d}=$ High & Codec $=$ MPEG -4 \\
\hline & $\mathrm{BER}=10^{-4}$ & Ideal TV Size $=30-40 ”$ \\
\hline & $\mathrm{SNR} \geq 5 \mathrm{~dB}$ & Resources used more than necessary \\
\hline & Capacity $\leq 75 \%$ & \\
\hline \multirow[t]{6}{*}{5} & Coverage area $=$ Very high & $\mathrm{Res} / \mathrm{fps}=720 / 25 \mathrm{i}$ \\
\hline & Separation distance $=$ Very low & Colour $=$ Rec709 \\
\hline & $\mathrm{d}=$ Low & Codec $=$ MPEG -4 \\
\hline & $\mathrm{BER}=10^{-4}$ & Ideal TV Size = 20-30" \\
\hline & $\mathrm{SNR} \geq 4 \mathrm{~dB}$ & Unacceptable resource usage \\
\hline & Capacity $>75 \%$ & \\
\hline \multirow[t]{6}{*}{6} & Coverage area $=$ Very high & \\
\hline & Separation distance $=$ Very Low & No video received \\
\hline & $\mathrm{d}=$ Very high & Video outage \\
\hline & $\mathrm{BER}=10^{-2}$ & Should not be allowed to happen \\
\hline & $\mathrm{SNR} \geq 20 \mathrm{~dB}$ & \\
\hline & Capacity $>75 \%$ & \\
\hline
\end{tabular}

* $\mathrm{d}=$ Distance between transmitter and receiver, Res/fps $=$ Resolution/frame rate

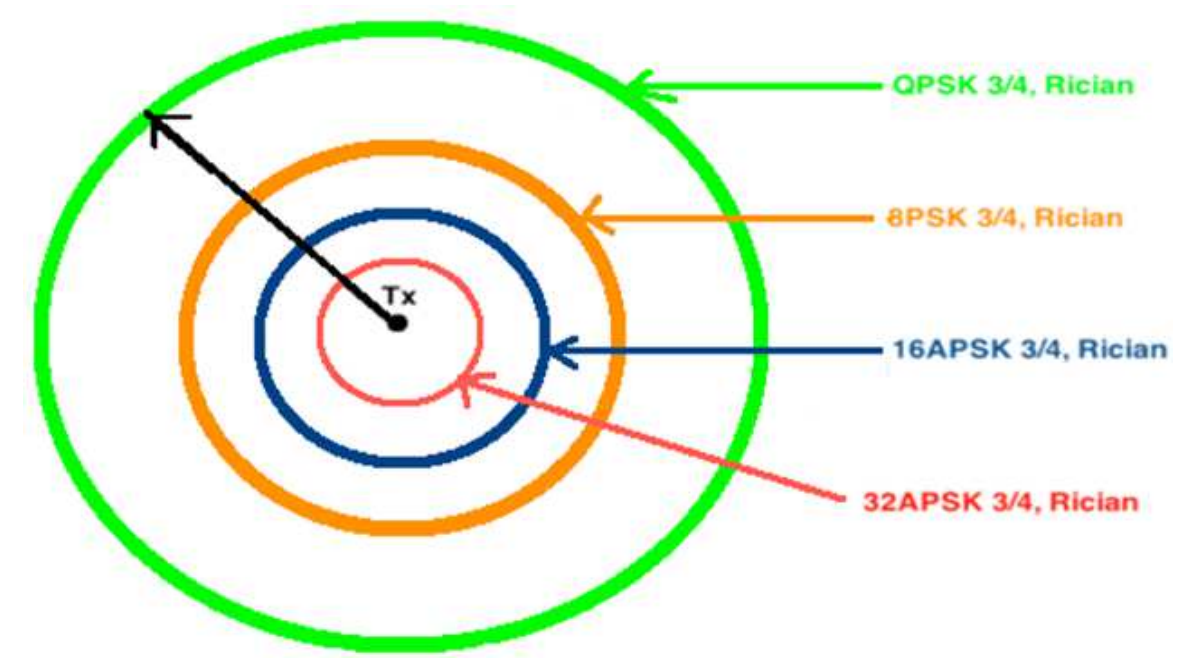

Fig. 8: Required distance between transmitter and receiver to maintain $3 \times 10-5$ BER based on Fig. 7 results (approximate depiction) 


\section{Conclusion}

In this study, experimental results obtained for BER vs. SNR for a UHD video signal, have been used to calculate the system capacity, spectral efficiency and the distance between transmitter and receiver. Using the results of these parameters, an adaptive video quality scenario is proposed for load balancing, using the principle of inclusion. Such a model will help in varying the video parameters as per the available resources, to prevent any kind of signal outage in the future broadcast scenario.

\section{Acknowledgement}

Victoria University colleagues that helped in the detailed discussion during the research period are greatly acknowledged.

\section{Author's Contributions}

Urvashi Pal: Has contributed towards the research plan development, problem formulation, experimental simulation, data analysis and writing of the manuscript.

Horace King: Has contributed towards the research plan development, problem formulation and data analysis and manuscript editing.

\section{Ethics}

Publication of this paper will not lead to any ethical issue.

\section{References}

Advanced Television, 2014. DVB approves UHDTV HEVC delivery profile.

Bettancourt, R.E. and H.M. Peha, 2015. On the trade-off between spectrum efficiency and transmission cost in traditional and SFN-based broadcast television. IEEE DySPAN.
DVB, 2005. User guidelines for the second generation system for broadcasting, interactive services, News Gathering and other broadband satellite applications (DVB-S2). Digital Video Broadcasting.

Eutelsat, 2015. Ultra HD Guidebook.

Intelsat, 2015. At the forefront of $4 \mathrm{~K}$ : Live, True $4 \mathrm{~K}$ ultra high definition television, end-to-end video transmission over satellite.

King, H. and U. Pal, 2015. A statistical approach to determine handover success using the principle of inclusion and load variation on links in wireless networks. IJICTA.

Kenichiro, M., T. Yamashita, Y. Nishida and M. Sugawara, 2014. Color management for wide-colorgamut UHDTV production. SMPTE Motion Imaging J., 124: 19-27. DOI: 10.5594/j18528

Limmer, C. and M. Chabrol, 2014. Ultra high definition market outlook and next steps.

Pal, U. and H. King, 2017. Cost increase due to UHD video broadcasting as compared to HD. SMPTE Conf.

Pal, U. and H. King, 2015a. DVB-S2 channel estimation and decoding in the presence of phase noise for nonlinear channels. IJICTA.

Pal, U. and H. King, 2015b. Effect of UHD HFR on video transmission. SMPTE Conference.

SES, 2013. Next generation of TV viewing: Developing the path for Ultra HD.

TT, 2013. The trade-off between image resolution and field of view: The influence of lens selection.

Vignelles, A. and D. Marshall, 2015. Broadcast in the age of disruption.

Wang, J. and H. Zhang, 2012. Capacity on $60 \mathrm{GHz}$ wireless communication system over fading channels. J. Networks. 